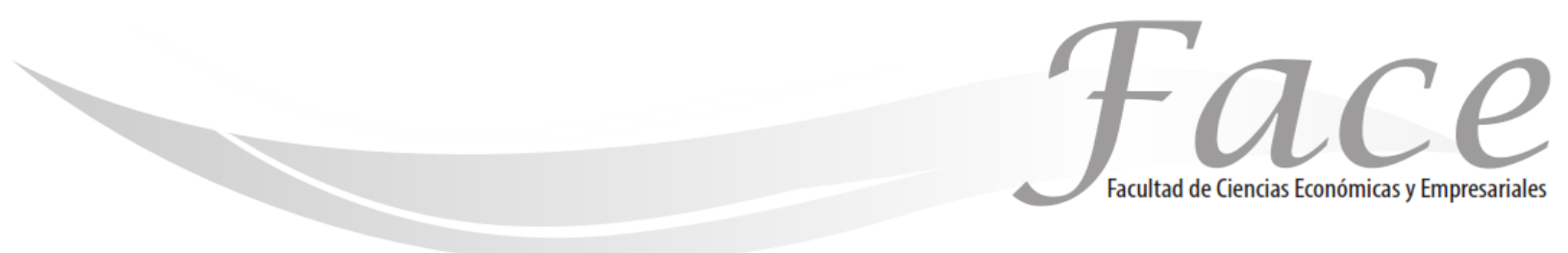

ISSN Impreso: 1794-9920

ISSN Electrónico: 2500-9338

Volumen $17-\mathrm{N}^{\circ} 1$

Año 2017

Págs. 19 - 34

\title{
LA COMERCIALIZACIÓN EN LAS MICROEMPRESAS DE LA LOCALIDAD DE SANTA FE EN LA CIUDAD DE BOGOTÁ*
}

Fecha de Recepción: 1 de Diciembre de 2016

Fecha de Aprobación: 10 de Marzo de 2017

\author{
Mónica Eugenia Peñalosa Otero** \\ Enlace ORCID: http://orcid.org/0000-0002-2208-9224 \\ Gloria Calderón Carmona *** \\ Enlace ORCID: http://orcid.org/0000-0002-2176-702X \\ Giovanny Fernando Benavides **** \\ Enlace ORCID: http://orcid.org/0000-0002-0767-760X
}

Resumen:

Siguiendo el compromiso de la Universidad Jorge Tadeo Lozano estipulado en el Proyecto Educativo Institucional, los esfuerzos de cada facultad y de cada programa académico están orientados hacia la investigación formativa y de rápida aplicación en la comunidad. De esta manera se pretende a través de este proyecto permear la realidad comercial y estratégica y originar un documento que sirva de enlace entre la academia y el sector real como aporte significativo para la solución de problemáticas sociales. Sin lugar a dudas, la Facultad de Ciencias Económicas y Administrativas, puede realizar importantes aportes en la medida que profundice en los papeles del productor y del consumidor y en los distintos ámbitos de aplicación del enfoque comercial. De igual manera, a través de su producción mejorar los indicadores en las mediciones de los grupos de investigación de la UJTL.

En función de las políticas de investigación institucionales, este proyecto atenderá de manera dinámica a las siguientes demandas: generación de opciones frente a las necesidades de formación básica en pregrado, consolidación del grupo de investigación del programa, aumento de los estudiantes inscritos en semilleros de investigación, creación de conocimiento y aplicación en la formación de estudiantes, incremento de la productividad académica de los docentes y fortalecimiento de las relaciones entre investigadores de la UJTL, entidades gubernamentales y grupos de investigación de otras entidades académicas.

Así mismo, dinamiza el campo de análisis y participación de los programas de Mercadeo, Contaduría Pública y Comercio Internacional, en el desarrollo de competencias y capacidades para contribuir con el fortalecimiento de los microempresarios de la localidad de Santa Fe

Palabras Claves: Comercialización, microempresas, marketing, contabilidad, logística.

* Artículo de reflexión resultado de la investigación titulada Propuesta de mejora de las prácticas comerciales aplicadas por los microempresarios de la localidad Santa Fe en la ciudad de Bogotá.

** Profesora Asociada de la Facultad de Ciencias Económico-Administrativas, Universidad Jorge Tadeo Lozano, Bogotá, Colombia. Magister en Mercadeo Agroindustrial. Profesional en Relaciones Internacionales. Investigadora Jr. de Colciencias. Se ha venido desempeñando en los últimos años como Docente - Investigadora en la Universidad Jorge Tadeo Lozano, la Universidad de La Salle y la Universidad de Pamplona - Colombia. Correo electrónico: monica.penalosa@utadeo.edu.co 
*** Profesora Asociada de la Facultad de Ciencias Económico-Administrativas, Universidad Jorge Tadeo Lozano, Bogotá, Colombia. Magíster en Administración de Instituciones Educativas del Instituto Tecnológico de Estudios Superiores de Monterrey. Coordinadora del Área Contabilidad e Información Contable. Desarrolla las asignaturas: Fundamentos de Contabilidad y Contabilidad y Análisis Financiero. Correo electrónico: gloria.calderon@utadeo.edu.co

*** Profesor Asociado de la Facultad de Ciencias Económico-Administrativas, Universidad Jorge Tadeo Lozano, Bogotá, Colombia. Magíster en Negocios Internacionales de la Univerté de Pau et des Pays de L’Adour, Francia. Especialista en Logística Comercial, Universidad Jorge Tadeo Lozano. Correo electrónico: giovanny.benavides@utadeo.edu.co

\title{
MARKETING IN THE MICROENTERPRISES OF THE SANTA FE LOCATION IN THE CITY OF BOGOTÁ
}

\begin{abstract}
Following the commitment of the Jorge Tadeo Lozano University stipulated in the Institutional Educational Project, the efforts of each faculty and each academic program are oriented towards formative research and of rapid application in the community. In this way, it is intended through this project to permeate the commercial and strategic reality and to create a document that serves as a link between academia and the real sector as a significant contribution to the solution of social problems. Undoubtedly, the Faculty of Economic and Administrative Sciences can make important contributions to the extent that it deepens the roles of the producer and the consumer and in the different fields of application of the commercial approach. Likewise, through its production improve the indicators in the measurements of the research groups of the UJTL.
\end{abstract}

Based on institutional research policies, this project will dynamically respond to the following demands: generation of options against basic training needs in undergraduate, consolidation of the research group of the program, increase of students enrolled in research seedlings, Creation of knowledge and application in the training of students, increase academic productivity of teachers and strengthening relationships between UJTL researchers, government entities and research groups from other academic entities.

It also stimulates the field of analysis and participation of the Marketing, Public Accounting and International Trade programs in the development of competencies and capacities to contribute to the strengthening of microentrepreneurs in the city of Santa Fe.

Keywords: Commercialization, microcompany, marketing, accounting, logistics.

\section{MARKETING NAS MICROEMPRESAS DA LOCALIZAÇÃO DE SANTA FE NA CIDADE DE BOGOTÁ}

\begin{abstract}
Resumo
Seguindo o compromisso da Universidade Jorge Tadeo Lozano estipulado no Projeto Educacional Institucional, os esforços de cada faculdade e cada programa acadêmico são orientados para pesquisa formativa e de aplicação rápida na comunidade. Desta forma, pretende-se que este projeto permeie a realidade comercial e estratégica e crie um documento que sirva de vínculo entre a academia e o setor real como um contributo significativo para a solução de problemas sociais. Sem dúvida, a Faculdade de Ciências Econômicas e Administrativas pode fazer contribuições importantes na medida em que aprofunda os papéis do produtor e do consumidor e nos diferentes campos de aplicação da abordagem comercial. Da mesma forma, através da sua produção, melhoram os indicadores nas medidas dos grupos de pesquisa da UJTL.

Com base em políticas de pesquisa institucional, este projeto responderá dinamicamente às seguintes demandas: geração de opções contra necessidades básicas de treinamento em graduação, consolidação do grupo de pesquisa do programa, aumento de alunos matriculados em mudas de pesquisa, criação de conhecimento e aplicação na Formação de estudantes, aumento da produtividade académica dos professores e fortalecimento das relações entre pesquisadores da UJTL, entidades governamentais e grupos de pesquisa de outras entidades acadêmicas.
\end{abstract}


Também estimula o campo de análise e participação dos programas de Marketing, Contabilidade e Comércio Internacional no desenvolvimento de competências e capacidades para contribuir para o fortalecimento de microempresários na cidade de Santa Fé.

Palavras-chave: Comercialização, microcompanhia, marketing, contabilidade, logística 


\section{INTRODUCCIÓN:}

La administración pública dimensiona su actuar en la construcción de una sociedad más colectivista y menos individualista, en este sentido las asociaciones públicas y privadas debería iniciarse en procesos participativos y de desarrollo de políticas generadoras de impacto en la dinámica empresarial, especialmente en aquellas zonas marginadas donde el factor empleo inicia a través de la informalidad.

En este sentido el papel de la universidad, en el actuar dentro del sector productivo deberá ser el de participar activamente de la mano de las líneas de investigación que trabajan; con ello se da cabida a la generación de espacios que permitan la discusión y la participación en el desarrollo social y empresarial de las microempresas de la cuidad de Bogotá.

De acuerdo a lo anterior, este artículo pretende analizar las prácticas comerciales aplicadas por los microempresarios de la localidad de Santa Fe en la cuidad de Bogotá; entendidas como el manejo que se está dando en el campo del marketing, la logística comercial y la convergencia contable. Lo anterior, teniendo en cuenta el número de comerciantes existentes y registrados ante la cámara de comercio de Bogotá y el impacto que tienen los mismos en el sector productivo, en la constante búsqueda de su desarrollo social y empresarial.

La participación desde la academia en el desarrollo de investigaciones en sectores productivos, contribuyen sin duda al fomento de capital humano y al fortalecimiento de la industria y su promoción; pues favorece la generación de capacidades y competencias gerenciales en los procesos de comercialización; así mismo, la contribución de capital intelectual genera la reducción de algunas dificultades latentes y se puede contribuir con el fortalecimiento social y empresarial.

\section{MARCO TEÓRICO:}

\subsection{Las Pymes}

La mayoría de las micro y pequeñas empresas colombianas tienen como su principal objetivo consolidarse en los mercados locales, nacionales e internacionales desarrollando el concepto de venta directa y fuerza de venta que dependen de locales propios y distribuidores independientes.

A nivel mundial, las Pymes se han convertido en principal fuente de desarrollo; en Colombia son generadoras de gran parte del empleo y la producción de bienes y servicios. Sin embargo, son ignoradas por su falta de competitividad frente a otras empresas existentes y posicionadas en el mercado. Una de las razones diferenciadoras podría ser la baja aplicación de prácticas comerciales en la dinámica de sus negocios, lo cual, las limita dentro de la dinámica de fomento y desarrollo del país.

Esta percepción ha ido cambiando durante los últimos gobiernos, quienes han trabajado en el establecimiento de políticas para promover el desarrollo de las Pymes a través de créditos y otras ayudas como capacitaciones, aun así siguen siendo crecientes las dificultades a las cuales se enfrentan las mismas.

El documento CONPES 3678 del 26 de julio de 2010, denominado Política de Transformación Productiva, un modelo de desarrollo sectorial para el país, propende por la generación de crecimiento sostenible en la economía y el empleo y busca desarrollar sectores altamente competitivos y generadores de valor agregado, alcanzando estándares de clase mundial. El objetivo de este documento CONPES es formular la Política de Transformación Productiva, mediante la consolidación de un modelo de desarrollo económico sectorial que guíe el crecimiento y genere bienestar para el país. La ejecución del plan de acción, marca el inicio de la transformación productiva del sector y se estructura con base en iniciativas agrupadas alrededor de cuatro ejes temáticos: el desarrollo de capital humano, la normatividad y regulación, el fortalecimiento de la industria y promoción y la infraestructura.

\subsection{El Marketing y sus Aplicaciones}

El Marketing consiste en actividades para facilitar y estimular intercambios dentro de un grupo de fuerzas externas dinámicas y cuya finalidad es planear, fijar el precio, promover y distribuir los productos satisfactores de necesidades y deseos entre los mercados metas hasta alcanzar objetivos corporativos". (Moreno, 2005, p 14).

El objeto del intercambio se llama genéricamente producto, el cual puede ser un bien, un servicio, una idea, persona o lugar que pueden ser intercambiadas. Es así, como el mercadeo y la publicidad se convierten en unas de las principales herramientas para la administración eficaz y eficiente de las empresas, asumiendo la innovación, anticipación y excelencia, como el reto para alcanzar el éxito.

Una vez identificadas las necesidades o preferencias, la empresa tiene que satisfacerlas, por lo tanto debe considerar los siguientes aspectos: 
- El producto: base definitiva para que el cliente determine si llena sus expectativas y sus necesidades quedan satisfechas, por lo que se debe considerar que la alternativa de solución iguale las necesidades tanto como sea posible.

- El sistema de entrega: poner oportunamente el producto al alcance del cliente.

- Concientizar al cliente de la disponibilidad y beneficios del producto: comunicación: convencerlo de comprarlo a través de la promoción.

- El producto debe tener el precio correcto para que el cliente pueda pagarlo y esté dispuesto a seleccionarlo sobre todos los ofertados.

La mezcla de mercadotecnia es el conjunto de estos cuatro aspectos las "4 P" de (McCarthy, 1984: p.46) producto, precio, plaza, promoción que no es más que la oferta completa que la empresa da a sus consumidores. Hoy en día es una práctica común en todo tipo de organizaciones plantear esfuerzos de mercadotecnia alrededor de estas 4 $\mathrm{P}$ que proporcionen al mercado la mayor satisfacción que la ofrecida por la competencia y evidencia resultados financieros atractivos a través de lo poderosa que sea esta mezcla. La mezcla de mercadeo representa la propuesta de valor la cual se presentará a los consumidores, clientes según sea el caso, en esta se definen El producto o servicio, el precio, la comunicación y la distribución.

El producto o servicio, o el intangible diferente al servicio. Aunque diferenciar los productos y servicios no es tan simple en las economías modernas, pues es difícil encontrar un producto sin ningún servicio asociado y de igual manera no es fácil que los servicios no tengan un tangible asociado al mismo, por lo cual se suele hablar más de ofertas complementarias (producto y servicio) a continuación se hace una breve diferencia. El producto es un tangible que es una conjunción de atributos que se le presenta al consumidor para resolver su necesidades y satisfacer sus deseos. El producto incluye unas características físicas y utilitarias que le permiten satisfacer las necesidades, pero también son atributos del producto, la marca, la etiqueta y el empaque, el servicio asociado al mismo, la garantía, la calidad y el diseño. El servicio es un intangible en general son "hechos actividades 0 rendimientos" (Hoffman, D. 2007) normalmente el valor del servicio se genera en la prestación misma, de igual manera tendrá marca, garantía y bienes asociados a su prestación.

El precio es un componente del valor percibido por los consumidores, una adecuada definición del precio y de la estrategia de precio garantizan mayores probabilidades de éxito que el no hacerlo.
La distribución es la variable encargada dentro de la mezcla de mercadeo de hacer llegar los productos terminados de la forma más adecuada a su consumidor final. Hay dos decisiones importantes en la distribución, la primera tiene que ver con la definición del tipo de distribución y la segunda con el tipo y la forma del canal de distribución.

Los tipos de distribución en marketing son tres:

- La distribución masiva o intensiva. Este tipo de distribución normalmente es la escogida para los bienes de conveniencia o de consumo masivo, bienes de precios relativamente bajos y de alta rotación, todos esos bienes que el consumidor considera que no vale la pena desplazarse largas distancias para adquirirlos y los compra de manera rápida y con procesos de compra simplificados y rápidos. En general el consumidor no se desgasta mucho comparando precios y marcas y remplaza de una manera relativamente fácil su marca preferida. Dentro de este tipo de productos encontramos las pilas, papas fritas, bombillos, gaseosas, galletas, etc.) Este tipo de bienes debe tener distribución intensiva, por lo tanto debe tener presencia en todos los puntos de venta posibles, tiendas, mini-mercados, supermercados e Hipermercados, normalmente puntos de venta poco o nada especializados.

- La distribución selectiva. Se realiza a través de varios puntos de venta pero no en todos los posibles como sucede con la distribución masiva, normalmente los bienes comparados (ropa, electrodomésticos, muebles, zapatos etc.) que se venden en tiendas especializadas, tienen este tipo de distribución. Los consumidores consideran que la compra de estos bienes es más importante y normalmente tiene un mayor nivel de involucramiento y por lo tanto realiza un proceso de compra más cuidadoso, comparando marcas y precios y desplazándose para buscar la mejor opción.

- $\quad$ La distribución exclusiva: Esta distribución se realiza a través de muy pocos puntos de venta, muchas veces incluso solamente a través de uno solo, el productor puede hacer un acuerdo con un distribuidor 0 puede el mismo generar un punto propio para vender los productos, estas tiendas especializadas en un tipo de producto y en una sola marca son utilizados para distribuidor bienes exclusivos. Marcas de ropa de diseñador, automóviles de alta gama, muebles exclusivos, son un ejemplo de los bienes que se venden con este tipo de distribución. 
Los canales de distribución son la otra decisión importante es la definición del canal de distribución, estos se entienden como las personas $u$ organizaciones que se encuentran entre el productor y el consumidor final y ayudan a llevar el producto de manera rápida y segura, cuando y como el consumidor lo necesita. La distribución puede usar un canal directo, en el cual el productor se encarga de vender directamente su producto al consumidor final sin hacer utilizar ningún mayorista o minorista. Sin embargo normalmente los productos se distribuyen de manera indirecta, por canales que incluyen mayoristas, minoristas y agentes comerciales.

La promoción y comunicación es el elemento de la mezcla de mercadeo que permite informar persuadir y recordarle al consumidor la existencia y las ventajas de los productos y servicios, buscando cambiar comportamientos de consumo. El estratega de mercadeo debe definir cuál será la mezcla de comunicación y promoción utilizada. De acuerdo al producto las organizaciones debe invertir sus recursos en una serie de herramientas de promoción y comunicación, las principales son las siguientes:

- Venta personal es una de las formas de promoción y comunicación más utilizada por muchas empresas, las cuales invierten sus recursos en una fuerza de venta que promociona, da a conocer y distribuye los productos, empresas como Herbalife, Yambal, Anway son ejemplos de organizaciones que emplean especialmente este tipo de promoción y comunicación.

- Las relaciones públicas son los esfuerzos realizados por las organizaciones para mejorar la imagen, con la sociedad y con su grupo objetivo de consumidores. Las herramientas de las relaciones públicas son muchas, el acercamiento a líderes de opinión, el desarrollo de publicaciones que generen relaciones con sus consumidores y usuarios, el material audiovisual de difusión, eventos de lanzamiento de productos o servicios nuevos, la participación o el desarrollo de eventos de obras benéficas, ruedas de prensa, publirreportajes, etc.

- La publicidad se entiende como la transmisión de un mensaje publicitario a través de un medio de comunicación masivo (radio, prensa, televisión, internet, revistas, vallas, etc), buscando influir sobre las preferencias de los consumidores y usuarios. Normalmente encontraremos cuatro tipos de publicidad, la de recuerdo, la persuasiva, la informativa o la de comparación.

- Las promociones comerciales son las acciones que realizan las organizaciones para facilitar el flujo de productos y servicios hacia el mercado, busca hacer más atractiva la propuesta de valor, dándole mayores beneficios al consumidor final o a los intermediarios. Dentro de las acciones más comunes de las promociones podemos encontrar los descuentos, los regalos automáticos, los concursos y los cupones de ahorro.

Vásquez, (2006) señala que la disciplina del Marketing aporta distintas formas de concebir y ejecutar la función comercial 0 relación de intercambio. Entre ellas se encuentra la filosofía de empresa, entendida como la actitud de concebir la relación de intercambio por parte de la empresa, donde la planeación y las operaciones van orientadas al cliente, considerando que él es lo primero, siempre tiene la razón y se convierte en el centro de las decisiones de la empresa, a pesar de ser un elemento externo e incontrolable. Es prioridad el consumidor para asegurar en el mediano y largo plazo el éxito de la empresa, donde partiendo de sus necesidades y deseos, todos los departamentos y empleados deben contribuir a su satisfacción del modo más beneficioso tanto, para el comprador como para la organización. Por su parte la actividad empresarial es el intercambio a través de la identificación, creación, desarrollo y servicio a la demanda, actividades deben coordinarse, buscando asegurar la relación entre la empresa y el cliente, procesar deseos y proponer productos que los satisfagan (con el área de producción), fijarles precios de acuerdo a sus posibilidades (con el área de contabilidad y finanzas), organizar su comercialización en el mercado (distribución), así como darlos a conocer a los clientes (publicidad y ventas). Finalmente, la orientación al cliente y coordinación para alcanzar los objetivos del desempeño organizacional, buscando volumen de ventas rentable para empresas lucrativas y el bienestar y mejoramiento del nivel de vida para empresas no lucrativas. Hoffman (2007) se refiere a la planeación estratégica como la misión, visión metas y objetivos en función del consumidor. Busca expresar de una forma clara y sistemática las opciones elegidas por la empresa para asegurar su desarrollo a medio y largo plazo, las cuales se deben traducir en decisiones y programas de acción.

\subsection{Prácticas de distribución al detal aplicadas por los comerciantes}

La concentración de puntos de venta donde los compradores realizan sus compras pertenecen a cadenas de retail las cuales poseen en su mayoría varios tipos de puntos de venta alrededor de un nicho seleccionado, estas cadenas son generalmente administradas por estructuras aún más grandes (Grupos, Sociedades, casas matrices) que controlan en gran medida el grueso del mercado a nivel global. La pertenencia de un comercio a una cadena 0 grupo le permite en sí de gozar de variadas sinergias, 
facilitando el desarrollo de su actividad y mejorando el servicio al cliente. Estas cadenas de retail tienen la particularidad de tener redes logísticas comunes para la distribución de productos lo que le permite en general manejar los flujos de mercancías desde su lugar de producción o almacenamiento hasta los puntos de venta final.

Las operaciones de distribución han aumentado en complejidad esto objeto de los modelos de tercerización, almacenamiento, aumento de referencias vendidas y a la disminución de los niveles de stock sobre el conjunto de la cadena de distribución, lo cual coloca la logística en el corazón de las estrategias de mercadeo y distribución de las empresas. El objetivo es lograr un comercio minorista que opere en espacios geográficos reducidos, que maneje una necesidad inmediata atendiendo sectores marginales de bajo poder adquisitivo como ciertas localidades bogotanas manteniendo una red de distribución común al conjunto de redes que abastecen los retails. Teniendo en cuenta esto, no se pretende restringir la información hacia la gran distribución alimentaria, pues existen otro tipo de especialistas zonales tipo tiendas de deportes, muebles, jardín, bricolaje, juguetería, textil y en general comercio al por mayor nominado en Colombia como "pajareras".

En este sentido, se hace un enfoque en la red de distribución que abastecen formatos múltiples desde la tienda de barrio hasta el hipermercado, pasando por supermercado, las cooperativas y la gran superficie especializada, agrupando el conjunto de actividades logísticas como un todo denominado gran distribución. El sector de alimentos es considerado quizás el más desarrollado y en donde la cadena de distribución se percibe más compleja, razón por la cual gran parte del estudio estará concentrado sobre la base de la distribución alimentaria y el comercio al detal sin dejar de lado el comercio mayorista el cual se sostiene sobre la base del modelo económico donde se abastece comprando grandes volúmenes de mercancías para luego venderlas en pequeñas cantidades, distinto al comercio minorista el cual no mantiene un contacto directo con el proveedor inicial y dispone de cadenas de distribución distintas.

El comercio minorista consiste en vender mercancías en el estado en el que son compradas es decir sin transformación (o después de transformaciones menores) generalmente a una clientela particular independiente de las cantidades vendidas. Esta actividad comercial puede necesitar de procesos de aprovisionamiento, entrega 0 incluso implantación de su actividad donde se encuentra el cliente (Muebles o electrodomésticos). La comercialización de productos generalmente comprende actividades sucesivas las cuales inicial por un comerciante denominado mayorista, seguido de una actividad de comercio al detal, sin embargo no todas las mercancías requieren de un comercio al detal.

Un comercio al detal se soporta sobre la base de cuatro pilares los cuales responden necesariamente a una estrategia de comercialización y venta, en realidad su negocio se soporta sobre un contacto directo con el cliente y sobre su pretensión real frente a su comercio, es así como el diseño de una estrategia que le permita tener un producto en óptimas condiciones con un nivel bajo de stock es decir también un contacto casi que a diario con su proveedor debe llegar a ser en enlace que le permita obtener un posicionamiento.

La pregunta que surge en cuanto al nivel de éxito que se tiene en un comercio al detal es si el manejo dado es el óptimo para llevar el negocio hacia una proyección mayor. Podríamos decir que apartándonos de las grandes marcas y los casos exitosos de franquicias los cuales han desarrollado una forma de proceder que maximiza sus inversiones al máximo, raros son los emprendedores que optimizan el pleno desarrollo de su comercio. La transformación de la informalidad hacia lo formal ha modificado las cifras de comercio al detal de manera considerable dirigiéndose hacia una competencia rapaz establecida en los sectores específicos de micro comercio. En este sentido las empresas de detal deben ser mucho más eficaces para conservar y aumentar su clientela y mantener un alto nivel de rentabilidad. Para lograr esto se debe maximizar la eficiencia en cada uno de los cuatro pilares que sostiene el comercio al detal. Estos pilares son:

- Conveniencia o Especialidad: La estrategia de una empresa por conveniencia consiste en tener los precios más bajos del mercado y convertirse en el más grande distribuidor. WALL-MARK, COSTCO son ejemplos de empresas por conveniencia. Por el lado de las empresas por especialidad trabajan ofreciéndole a un cliente conocimientos especializados con productos exclusivos y muy selectos. Por ejemplo "the Wine Store" es reconocido en el mundo de los vinos y las catas en Bogotá y una referencia en este campo. En este sentido la orientación en la estrategia depende del modelo de negocio que se contemple y el objetivo será el de mejorar la posición del comercio al detal ya sea por conveniencia 0 por especialidad. Dicho de otra manera si se opta por una empresa por conveniencia se debe estar atento a cualquier oportunidad que permita reducir costos, ya sea desde lo laboral hasta el manejo con los proveedores. Si la estrategia se dirige hacia la especialidad se debe asegurar un nivel de capacitación alto con el objetivo de obtener una experticia marcada en el servicio y de igual manera en los productos. 
- Ubicación: la visibilidad que tenga un comercio al detal es esencial para el desarrollo de su actividad, centros de comercio, vías principales o secundarias se convierten en verdaderas plataformas de distribución, cuando el consumidor interpreta la existencia de nuestro negocio, no tenemos si no un segundo para transmitirle nuestra estrategia de venta. Para esto es suficiente realizar un simple ejercicio de encuestas dentro de la población urbana cerca al comercio minorista, en ella se deberá preguntar la existencia de un producto el cual por recordación deberá ser el negocio en cuestión. Si no existe recordación alguna se deberá de forma urgente realizar una estrategia comercial que corrija el error.

Algunos elementos dirigidos a mejorar la recordación basado en la ubicación:

- Uso de avisos más explícitos y llamativos de sus productos y/o servicios.

- Uso de activación de marca en punto de venta a través de disfraces o mascotas que estén en relación con el producto que se vende

- Uso de afiches publicitarios en el andén o alrededores

- Degustaciones o muestreo en el entorno del negocio

El objetivo es hacerse ver lo máximo posible tanto por el automovilista como por el peatón.

- $\quad$ Atención al cliente: la teoría de las 7P establece que los perceptibles son pieza fundamental de la estrategia comercial, el cliente concentra su decisión fundamentalmente en las emociones las cuales mantiene durante un largo tiempo y marcan su poder de decisión en una compra; si estas son negativas 0 neutras frente a un comercio minorista no ayudaran de ninguna manera la atención de otros potenciales clientes. En cambio, si la experiencia lograda a través de la atención o del producto ofrecido seguramente regresara y realizara un excelente mercadeo voz a voz. El mejor vendedor es el cliente directo o cliente habitual y para mantenerlo como esta figura se deberá encontrar la manera de hacerle sentir su vocación hacia él y esto cada vez que visite el establecimiento.

Una estrategia solida con el uso de cada uno de estos pilares hará un estrategia de comercialización mucho más eficaz que acompañada de una estrategia de distribución conectado proveedores con el empresario minorista y posteriormente con el cliente final identificara el negocio rápidamente el negocio en espacios geográficos reducidos atendiendo así un consumidor que demanda rapidez, precio y calidad. De esta menara el uso de herramientas contables, de mercadeo y de distribución se complementan y determinar el rumbo que puede requerir un empresario minorista.

- Mercadeo: El mercadeo consiste en un proceso administrativo y social gracias al cual determinados grupos 0 individuos obtienen lo que necesitan 0 desean a través del intercambio de productos 0 servicios.

\subsection{El reto de la convergencia contable}

La entrada en vigencia de las Normas Internacionales de Información Financiera (NIIF) en Latinoamérica ha estado marcada por diferentes experiencias; en algunos países han hecho adopción total de las NIIF, por ejemplo, Panamá, El Salvador y Paraguay. Otros lo han hecho de forma selectiva, como Perú, Ecuador, Costa Rica, Uruguay y Honduras (Manzano, 2005). En el año 2009, con la expedición de la Ley 1314, Colombia decide emprender el proceso de convergencia a las NIIF, sin embargo, la implementación, que es la etapa crítica de este proceso apenas está dando sus primeros pasos.

La adopción de las NIIF se ha difundido como un avance para reducir los costos de información entre los países y, por lo tanto, animar al comercio internacional de bienes y la inversión. Los estudios desarrollados sobre esta implementación suelen demostrar dificultades en el proceso de adopción y convergencia, pero también todos los efectos positivos producto de la transición a las nuevas normas.

Dichos problemas se originan por la complejidad del proceso, el cual requiere de conocimientos de otras disciplinas en las áreas de valorización de activos, instrumentos financieros, cálculos actuariales, riesgo, entre otros (Gómez et al., 2011).

Dentro de los retos que enfrentan las compañías se encuentra la definición de políticas contables de acuerdo con las NIIF, lo cual supone un conocimiento de parte de los diferentes miembros de la compañía, el equipo directivo y demás empleados involucrados con la elaboración de la información financiera, entendiendo que ellas deben ser establecidas por la alta dirección como los preceptos fundamentales a partir de los cuales se prepararán y presentarán, en adelante, los estados financieros. Así mismo, una entidad que adopta por primera vez debe reconocer los ajustes en las ganancias acumuladas y explicar el efecto de la transición a las NIIF en su posición financiera, rendimiento financiero y los flujos de efectivo, este será el primer efecto de las decisiones de la gerencia sobre las políticas contables (Gornik-Tomaszewski, 2010). 
Consecuentemente, será la fuente de evidencia para medir los primeros impactos de la convergencia.

Con el Direccionamiento estratégico del proceso de convergencia de las normas de contabilidad e información financiera y de aseguramiento de la información con estándares internacionales, expedido por el Consejo Técnico de la Contaduría Pública (2012) se orienta el desarrollo del proceso de convergencia y se da alcance al Direccionamiento que se había divulgado en el año 2011, todo como parte de la responsabilidad que por mandato de la Ley 1314 de 2009 recae sobre este organismo como la autoridad colombiana de normalización técnica de las normas contables de información financiera y de aseguramiento de la información.

En dicho Direccionamiento se incluye, entre otros, una clasificación de las normas entre aquellas relacionadas con la información financiera, NIF, las normas de aseguramiento de la información, NAl y las otras normas de información financiera, ONI. Además se ajusta la descripción de los grupos de usuarios que deben adelantar el proceso de implementación y las normas que les son aplicables, tal como se aprecia en la siguiente tabla tomada del párrafo 49 del Direccionamiento (CTCP, 2012):

\begin{tabular}{|c|c|c|c|}
\hline TIPO DE NORMAS & GRUPO 1 & GRUPO 2* & GRUPO $3^{*}$ \\
\hline & $\begin{array}{l}\text { a) Emisores de valores; } \\
\text { b) Entidades de interés público; } \\
\text { c) Entidades que tengan Activos totales superiores a } \\
\text { treinta mil ( } 30.000 \text { ) SMMLV o planta de personal } \\
\text { superior a doscientos (200) trabajadores; que no } \\
\text { sean emisores de valores ni entidades de interés } \\
\text { público y que cumplan además cualquiera de los } \\
\text { siguientes requisitos: } \\
\text { i. ser subordinada o sucursal de una compañia } \\
\text { extranjera que aplique NIIF; } \\
\text { ii. ser subordinada o matriz de una compania } \\
\text { nacional que deba aplicar NIIF; } \\
\text { iii. realizar importaciones (pagos al exterior, si se } \\
\text { trata de una empresa de servicios) o exportaciones } \\
\text { (ingresos del exterior, si se trata de una empresa de } \\
\text { servicios) que representen más del 50\% de las } \\
\text { compras (gastos y costos, si se trata de una empresa } \\
\text { de servicios) o de las ventas (ingresos, si se trata de } \\
\text { una compan̂ia de servicios), respectivamente, del } \\
\text { año inmediatamente anterior al ejercicio sobre el } \\
\text { que se informa, o } \\
\text { iv. ser matriz, asociada o negocio conjunto de una o } \\
\text { más entidades extranjeras que apliquen NIIF. }\end{array}$ & $\begin{array}{l}\text { a) Empres as que no cumplan con los } \\
\text { requisitos del literal c) del grupo 1; } \\
\text { b) Empresas que tengan Activos totales } \\
\text { por valor entre quinientos ( } 500 \text { ) y } \\
\text { treinta mil ( } 30.000 \text { ) SMMLV o planta de } \\
\text { personal entre once (11) y doscientos } \\
\text { (200) trabajadores, y que no sean } \\
\text { emisores de valores ni entidades de } \\
\text { interés público; y } \\
\text { c) Microempres as que tengan Activos } \\
\text { totales excluida la vivienda por un } \\
\text { valor máximo de quinientos (500) } \\
\text { SMMLv o Planta de personal no } \\
\text { superior a los diez (10) trabajadores, } \\
\text { y cuyos ingresos brutos anuales sean } \\
\text { iguales o superiores a } 6.000 \text { SMMLV. } \\
\text { Dichos ingresos brutos son los } \\
\text { ingresos correspondientes al afio } \\
\text { inmediatamente anterior al periodo } \\
\text { sobre el que se informa. } \\
\text { Para la clasificación de aquellas } \\
\text { empres as que presenten } \\
\text { combinaciones de parámetros de } \\
\text { planta de personal y activos totales } \\
\text { diferentes a los indicados, el factor } \\
\text { determinante para dicho efecto, será el } \\
\text { de activos totales. }\end{array}$ & $\begin{array}{l}\text { (a) Personas naturales o } \\
\text { juridicas que cumplan los } \\
\text { criterios establecidos en el } \\
\text { art. } 499 \text { del Estatuto } \\
\text { Tributario (ET) y normas } \\
\text { posteriores que lo modifiquen. } \\
\text { Para el efecto, se tomará el } \\
\text { equivalente a UVT, en salarios } \\
\text { mínimos legales vigentes. } \\
\text { (b) Microempresas que tengan } \\
\text { Activos totales excluida la } \\
\text { vivienda por un valor máximo } \\
\text { de quinientos (500) SMMLV o } \\
\text { Planta de personal no } \\
\text { superior a los diez (10) } \\
\text { trabajadores que no cumplan } \\
\text { con los requisitos para ser } \\
\text { incluidas en el grupo } 2 \text { ni en el } \\
\text { literal anterior. }\end{array}$ \\
\hline \begin{tabular}{|l|} 
Normas de Información \\
Financiera - NIF
\end{tabular} & NIIF plenas (IFRS) & NIIF para PYMES (IFRS for SMEs) & \multirow{2}{*}{$\begin{array}{l}\text { E Gobierno autorizará de } \\
\text { manera general que ciertos } \\
\text { obligados lleven contabilidad } \\
\text { simplificada, emitan estados } \\
\text { financieros y revelaciones } \\
\text { abreviados o que estos sean } \\
\text { objeto de aseguramiento de la } \\
\text { información de nivel } \\
\text { moderado }\end{array}$} \\
\hline $\begin{array}{l}\text { Normas de } \\
\text { aseguramiento de la } \\
\text { Información NAl }\end{array}$ & \multicolumn{2}{|c|}{$\begin{array}{l}\text { 1) Estándares Internacionales de Auditoría (ISA - por sus siglas en inglés), } \\
\text { 2) Estándares Internacionales sobre Trabajos de Revisión Limitada (ISRE - por sus } \\
\text { siglas en inglés), 3) Estándares Internacionales sobre Trabajos de Aseguramiento } \\
\text { (ISAE - por sus siglas en inglés), 4) Estándares Internacionales sobre Servicios } \\
\text { Relacionados (ISRS - por sus siglas en inglés), 5) Estándares Internacionales de } \\
\text { Control de Calidad para los servicios incluidos en los estándares de IAASB (ISQC - } \\
\text { por sus siglas en inglés), 6) Estándares Internacionales sobre Prácticas de Auditoría } \\
\text { (IAPS-por sus siglas en inglés) y 7) Código de Ética para Contadores Profesionales } \\
\text { (IESBA -por sus siglas en inglés). }\end{array}$} & \\
\hline \begin{tabular}{|l|} 
Otras Normas de \\
Información Financiera - \\
ONI
\end{tabular} & \multicolumn{3}{|c|}{$\begin{array}{l}\text { Desarrollo normativo de manera general o para cada grupo de todo lo relacionado con el sistema documental } \\
\text { contable, registro electrónico de libros, depósito electrónico de información, reporte de información mediante } \\
\text { XBRL, y los demás aspectos relacionados que sean necesarios. }\end{array}$} \\
\hline
\end{tabular}

"Nota: Las empresas del grupo 2 y del grupo 3 que deseen voluntariamente aplicar las NIIF plenas (IFRS), o para el grupo 3 la NIIF para PYMES podrán hacerlo teniendo en cuenta las obligaciones que de dicha aplicación se derivarán (ver párrafo 58).

En desarrollo del reto que impone el proceso de convergencia, instituciones de control como la Superintendencia de Sociedades y la Superintendencia Financiera de Colombia han sido vitales para impulsarlo. Se han expedido, entre otras, disposiciones como la Circular Externa de Supersociedades 115-000001 del 09 de enero de 2013, con el propósito de que las empresas clasificadas en el grupo 1 preparen un plan de implementación de las NIIF (Superintendencia de Sociedades, 2013) y la 115-00002 del 14 de marzo de 2012 para las microempresas que amplía el marco de 
referencia para el entendimiento y la aplicación de las Normas (Superintendencia de Sociedades, 2012).

Las normas dictadas por todos los organismos (Congreso, ministerios, Consejo Técnico de la Contaduría Pública, superintendencias y demás entes competentes y relacionados) denotan, al mismo tiempo, avances y dificultades. Avances, en la medida en que pone a Colombia a dialogar en el mismo lenguaje que un sinnúmero de países con los cuales tiene relaciones de todo tipo y que hacen necesario el lenguaje contable común. Dificultades, en tanto la aplicación de la normativa, de inmediato, pone a las empresas en trance de hacer modificaciones sustanciales en todas sus instancias organizacionales pues se trata de un cambio que afecta transversalmente los sistemas internos de información de las empresas.

La adopción por primera vez, eleva los costos de proceso, producción y análisis de la información. La tecnología necesaria o su adaptación, las políticas internas, los manuales y planes de cuentas contables, la capacitación del personal, no solo el contable sino el de toda la compañía, los planes de auditoría externa y la comunicación a los usuarios de la información financiera. Se elevan o aparecen, también, los costos de consultoría externa en materias contable, legal, tributaria, de actuaría, de peritaje, etc. La administración empresarial debe, por lo tanto, hacer grandes esfuerzos para hacer un tránsito hacia las NIC-NIIF, la planeación del proceso de convergencia debe contemplar tanto los planes de tipo contable como los planes de recursos requeridos, fijándose metas y propósitos claros para obtener el máximo de provecho y minimizar los traumas durante el cambio y en el periodo de adopción plena.

Al mismo tiempo, siendo que las normas son las mismas para todos los de cada grupo, ponerlas en práctica no implica idénticas transformaciones, adaptaciones 0 dificultades pues las estructuras de balance son diferentes así se trate de empresas de un mismo sector o industria y su aplicación obliga a la administración a formular toda clase de estrategias para poner a punto tanto la parte puramente formal y mecánica como las políticas contables propiamente dichas, todo de acuerdo, como se anotó, con el caso específico de la empresa que hace la adopción. Hay una gran dificultad en las empresas que tienen negocios y filiales con empresas del exterior. La obligación de armonizar las normas con las empresas relacionadas (filiales, por ejemplo) con sede en otros países que tienen diferencias en los procesos de convergencia es una dificultad que para superarla, requiere del acopio de creatividad y conocimiento. En algunos países tienen NIIF plenas y en otros tienen normas híbridas $u$ otras formas de aplicación.
Para el caso de las pequeñas y medianas empresas fue expedido el decreto 3022 de 2013 por medio del cual se establecieron las condiciones para pertenecer a dicha categoría y ubicarse en el grupo 2, el decreto fijó el marco técnico normativo que detalla la aplicación de las NIIF para Pymes, este detalle corresponde a los estándares aprobados en el año 2009 por IASB y que actualmente es objeto de revisión para un ajuste en tiempo cercano. En el caso de las microempresas la dificultad para entrar en este proceso de convergencia guarda relación principalmente con la confusión que existe para los empresarios entre las responsabilidades inherentes a su negocio en materia contable independiente de aquellas en materia fiscal. Si bien el Código de Comercio establece, entre las obligaciones de los comerciantes, la de llevar contabilidad formal de sus negocios (Código de Comercio, Artículo 19), en nuestro medio es muy generalizado el hecho de que impere la norma fiscal, que para este caso asigna a las empresas del llamado Régimen Simplificado la obligación de manejar un libro fiscal de registro de operaciones diarias (Estatuto Tributario, Artículo 616), ocasionando con esto una distorsión en el imaginario de los comerciantes que consideran el citado libro fiscal como el sustituto de la contabilidad.

Para reglamentar lo relacionado con el nuevo manejo de la contabilidad en las microempresas enfrentando el reto de la convergencia contable, se expidió el Decreto 2706 de 2012 que consagra el marco técnico normativo de información financiera para las microempresas, este decreto fue modificado parcialmente por el Decreto 3019 de 2013 en lo que se refiere a la definición y características de las microempresas a quienes les es aplicable dicho marco técnico normativo. Aunque es una norma local, está fundamentada en las NIIF para Pymes y recoge elementos importantes del Decreto 2649 de 1993 y de otros estudios relacionados para detallar lo que se conocerá en adelante como Contabilidad Simplificada. Posteriormente con la Circular Externa 115-000003 del 14 de marzo de 2013, la Superintendencia de Sociedades fijó las pautas para el proceso de implementación, adicionada con una guía que permite a las microempresas tener parámetros y lineamientos generales para adelantarla.

\section{METODOLOGÍA:}

En una primera fase se hizo un estudio de fuentes secundarias para la revisión documental en lo relacionado con estudios previos en instituciones tales como Acopi y Cámara de Comercio de Bogotá, entre otras, a su vez se tomaron como referencia otras investigaciones construidas en torno al tema. En una segunda fase, para obtener la información con respecto a las prácticas aplicadas, se realizó una investigación de alcance cualitativo, con un 
enfoque exploratorio, puesto que es la herramienta más eficaz para poder comprender este fenómeno desde la propia perspectiva de los microempresarios de la localidad de Santa Fe.

Técnicas y Target: La técnica utilizada en este estudio fue la entrevista en profundidad. El target fue el de los microempresarios de la localidad de Santa Fe registrados en las bases de datos de la Cámara de Comercio de Bogotá, pertenecientes al barrio Las Nieves, de estratos socioeconómicos 2 y 3 , en edades comprendidas entre los 18 y 65 años.

Muestra: Se desarrollaron 30 entrevistas semi estructuradas, para la implementación de la prueba actitudinal sobre prácticas comerciales empleadas por los microempresarios de la localidad de Santa Fe, en personas de 18-65 años, pertenecientes al barrio Las Nieves, de estrato 2 y 3 . Muestreo: Para el estudio se tuvo en cuenta un muestro no probabilístico por conveniencia.

\section{RESULTADOS Y DISCUSIÓN:}

\section{Caracterización de las empresas en la localidad de Santafé}

De acuerdo con la cámara de comercio de Bogotá, en el año 2006 se registraron 227 mil empresas en Bogotá. El $5 \%$, equivalente a 11.252 empresas, se ubicaron en la localidad de Santafé. En comparación con las demás localidades de la ciudad, Santafé ocupó el noveno lugar por el número de empresas. En términos del valor de los activos, las empresas reportaron \$332 billones en Bogotá y $\$ 125$ billones en Santafé (38\%). Es decir, ocupó el primer puesto en este indicador.

Cuadro 1. Número de empresas matriculadas y renovadas en la localidad Santafé.

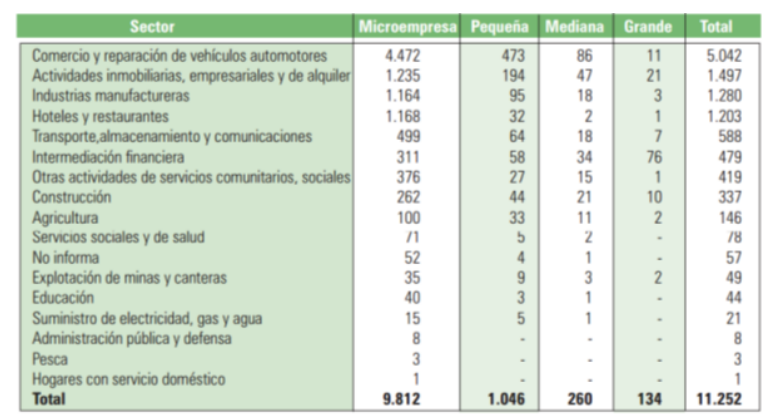

Fuentes: CCB (2006). Registro Mercantil, Cámara de Comercio de Bogotá. Proceso: Dirección de Estudios e Investigaciones de la CCB.
La localidad de Santafé cuenta con una estructura empresarial concentrada con el $83 \%$, en el sector de los servicios; con el $11 \%$ la industria y con el $3 \%$ la construcción. La mayor participación de los servicios se explica por la presencia de empresas registradas en la actividad comercial (45\%), que es el eje del mercado local. En menor medida, están las actividades que prestan las empresas inmobiliarias, empresariales y de alquiler (13\%) y los hoteles y restaurantes (11\%). Según el tamaño de las empresas, se puede afirmar que Santafé es una localidad de microempresarios. Del total de empresas en el 2006, se encontró un total de 9.812 microempresas, que representaron el $87,2 \%$ de las empresas establecidas en la localidad y el $5 \%$ de Bogotá. Las pymes representaron el $11,6 \%$, mientras la gran empresa registró el 1,2\%, participación relativamente baja.

En Santafé, se encuentran empresas que contribuyen con el desarrollo de la actividad económica y financiera. Se destacan: Grupo Aval Acciones y Valores, S.A., Dminegocios,S.C.A., Actiunidos, S.A., en actividades inmobiliarias, empresariales y de alquiler; Banco de Bogotá, S.A., Banco Agrario de Colombia, S.A., Granbanco, S.A., Banco Popular, S.A., Colpatria Red Multibanca, Banco Comercial Av Villas S A, Fondo Nacional de Ahorro, en la actividad de intermediación financiera y Ecopetrol, S. A., en el sector de explotación de minas y canteras. La mayoría de las empresas de Santafé se localizaron geográficamente en la parte centro occidental de la localidad, cerca de sus avenidas principales.

Los barrios que se destacaron por su concentración empresarial son: La Perseverancia, La Capuchina, Las Nieves, Santa Inés, La Alameda, San Diego, Samper, Sagrado Corazón, San Martín.

Así mismo, en la UPZ Las Nieves se encontró el mayor número de empresas, le siguen las UPZ Sagrado Corazón, Las Cruces, La Macarena y Lourdes. (Ver figura 2.1). Para abril de 2007, se encontraba reglamentada por el DAPD la UPZ Lourdes. La actividad de ocupó el primer lugar por número de empresas en la estructura empresarial de Santafé.

En el 2006, esta actividad obtuvo ventas por valor de $\$ 1.8$ billones, el $6 \%$ del total de las ventas locales, reportó un valor de activos de $\$ 1.2$ billones, el $1 \%$ del total de la localidad y ocupó al $10 \%$ del personal que contrataron las empresas de la localidad. Las actividades del sector comercio en Santafé se destacaron por su número y por el valor de sus activos. Por número, se destacaron las empresas de los subsectores así: el 36\%, comercio al por menor de productos nuevos de consumo doméstico en establecimientos especializados; el 19\%, comercio al por 
menor de otros nuevos productos de consumo, en establecimientos especializados; el 15\%, comercio al por menor, en establecimientos no especializados con surtido compuesto principalmente de alimentos, bebidas y tabaco y el $8 \%$, comercio al por mayor de productos de uso doméstico.

Las empresas dedicadas al comercio al por menor de productos nuevos de consumo doméstico en establecimientos especializados se concentraron en los barrios Santa Inés, La Capuchina, La Veracruz y Las Nieves. Las empresas dedicadas al comercio al por menor de otros nuevos productos de consumo, en establecimientos especializados, se concentraron en los barrios La Veracruz, La Capuchina, Las Nieves: mientras que las empresas dedicadas al comercio al por menor en establecimientos no especializados con surtido compuesto principalmente de alimentos (víveres en general), bebidas y tabaco se concentraron en los barrios Santa Inés, La Veracruz, Las Nieves, San Bernardo, La Capuchina y las Cruces; y las empresas dedicadas al comercio al por mayor de productos de uso doméstico se concentraron en los barrios Santa Inés y La Capuchina.

Según el valor de los activos, que permiten caracterizar la magnitud y concentración de la inversión empresarial, se destacaron por su participación en este indicador los subsectores de: el 17\%, comercio al por menor de otros nuevos productos de consumo, en establecimientos especializados; el $16 \%$, comercio al por mayor de productos de uso doméstico; $16 \%$, comercio al por menor de productos nuevos de consumo doméstico en establecimientos especializados y el $13 \%$, comercio al por mayor de materias primas agropecuarias; animales vivos; alimentos, bebidas y tabaco.

La actividad comercial en Santafé se caracterizó por su concentración por número de empresas y de activos en los subsectores dedicados al comercio al por menor de productos de uso doméstico y de distribución de alimentos, bebidas y tabaco, que se realiza en el mayor porcentaje a través de microempresas.

La segunda actividad que se destacó en la estructura empresarial en Santafé fue la actividad inmobiliaria, empresarial y de alquiler que concentró el 13\% de las empresas y el $15 \%$ del valor de los activos registrados por los empresarios. En el año 2006, esta actividad obtuvo ventas que representaron el $4 \%$ del total de la localidad y participó con el $17 \%$ del personal ocupado por las empresas inmobiliarias. Por número de empresas dedicadas a esta actividad, corresponde: el 36\%, actividades jurídicas y de contabilidad, teneduría de libros y auditoría; el 17\%, actividades empresariales (obtención y suministro de personal, seguridad, limpieza de edificios); el $16 \%$, actividades inmobiliarias realizadas con bienes propios o arrendados y el $6 \%$, actividades inmobiliarias realizadas a cambio de una retribución o por contrata.

En los barrios La Veracruz, Samper y Sagrado Corazón, se ubicaron las empresas dedicadas a las actividades jurídicas y de contabilidad, teneduría de libros y auditoría; asesoría en materia de impuestos, estudio de mercados y realización de encuestas de opinión pública; asesoría empresarial y en materia de gestión; en los barrios La Veracruz, Las Nieves, San Diego y la Capuchina, las empresas dedicadas a las actividades empresariales (obtención y suministro de personal, actividades de investigación y seguridad, limpieza de edificios, fotografía, envase y empaque); en los barrios La Veracruz, Santa Inés, La Capuchina, Sagrado Corazón, Las Nieves, San Diego y San Martín, las empresas dedicadas a las actividades inmobiliarias realizadas con bienes propios 0 arrendados (arrendamiento, urbanización, subdivisión, venta, alquiler).

Según la concentración del valor de los activos en la actividad inmobiliaria, empresarial y de alquiler, se destacó el subsector de actividades empresariales (obtención y suministro de personal, actividades de investigación y seguridad, limpieza de edificios, fotografía, envase y empaque), registró el $98 \%$ de los activos de las empresas de esta actividad. Los siguientes subsectores representativos en este indicador fueron: el 1\%, actividades inmobiliarias realizadas con bienes propios 0 arrendados y el $0,5 \%$, actividades jurídicas y de contabilidad. La industria es la tercera actividad representativa y dinámica de la economía local. En el 2006, se registró un total de 1.280 empresas, el $11 \%$ de las empresas locales. El $91 \%$ fueron microempresas. Las empresas industriales en Santafé reportaron activos por valor de $\$ 287$ mil millones, el $0,2 \%$ del total de activos de las empresas locales, obtuvo ventas por un valor aproximado de $\$ 279$ mil millones, el $1 \%$ del total local y contrató a 3.351 personas, el $5 \%$ del personal ocupado por los sectores económicos de la localidad.

En la industria manufacturera de Santafé, se identificaron 1.280 empresas, agrupadas en subsectores así: el 30\%, fabricación de prendas de vestir, preparado y teñido de pieles; el $7 \%$, actividades de edición; el $6 \%$, actividades de impresión; el 6\%, actividades de servicios relacionadas con las de impresión y el $6 \%$, industrias manufactureras (6\%).

En los barrios la Capuchina y Santa Inés, se ubicaron las empresas dedicadas a la industria de prendas de vestir (excepto ropa de cuero) (ropa exterior e interior para hombres, mujeres y niños, corsetería, camisería, vestidos de baño, ropa sobre medidas, servicios satélites; en los barrios La Veracruz, Las Nieves y la Capuchina, las actividades de edición y en los barrios La Veracruz, Las Nieves, La Capuchina y la Alameda, las actividades de impresión, periódicos, artículos escolares y de oficina e 
Mónica Eugenia Peñalosa Otero - Gloria Calderón Carmona - Giovanny Fernando Benavides

impresión. Según la clasificación por sector económico, el valor de los activos se concentró así: el $22 \%$, actividades de edición, la más representativa; el 17\%, fabricación de vidrio y de productos de vidrio; el $16 \%$, fabricación de productos minerales no metálicos ncp (cerámica no refractiva, cerámica refractaria, arcilla, cemento, cal, yeso, hormigón, para uso estructural) y el $12 \%$, fabricación de prendas de vestir (excepto ropa de cuero).

La actividad de hoteles y restaurantes es la cuarta por número de empresas en la localidad con el $11 \%$ de las empresas existentes en la localidad. En el 2006, el sector representó el $0,2 \%$ del valor de los activos registrados en la localidad. Ocupó al $2 \%$ del personal reportado por las firmas y obtuvo ventas que alcanzaron los $\$ 120 \mathrm{mil}$ millones, el $0,2 \%$ de las reportadas en ese año por los empresarios de la localidad. En la actividad de hoteles y restaurantes, se destacan por su número los siguientes subsectores: el $42 \%$, expendio a la mesa de comidas preparadas, en restaurantes; el $21 \%$, expendio a la mesa de comidas preparadas, en cafeterías; el $15 \%$, expendio de bebidas alcohólicas para el consumo en el establecimiento y el $8 \%$ alojamiento en residencias, moteles y amoblados.

En esta actividad, el $90 \%$ del valor de los activos registrados por los empresarios está en el subsector de expendio a la mesa de comidas preparadas, en restaurantes y el $9 \%$, en alojamiento en hoteles, hostales y aparta hoteles. Las empresas dedicadas al expendio a la mesa de comidas preparadas en restaurantes, se ubicaron en los barrios la Veracruz, Las Nieves, San Diego y La Capuchina; las empresas dedicadas al expendio a la mesa de comidas preparadas en cafeterías, se concentraron en los barrios Las Nieves, La Veracruz, La Capuchina y La Alameda; las empresas dedicadas al expendio de bebidas alcohólicas para el consumo en el establecimiento se concentraron, en los barrios Las Nieves, La Veracruz, La Alameda y San Bernardo; las empresas dedicadas alojamiento en residencias, moteles y amoblados, se concentraron en los barrios La Alameda, San Bernardo y La Capuchina.

\section{Tamaño de las empresas}

En el 2006, el valor de los activos de las empresas matriculadas y renovadas se concentró en las empresas de gran tamaño. Es decir, 134 empresas desarrollan su actividad mercantil con el $98,1 \%$ de los activos. Con una menor participación, están la pyme con 1.306 empresas y con el 1,5\% de los activos. La microempresa con 9.812 unidades productivas tiene el $0.1 \%$ de los activos. Por sector económico, el valor de los activos se concentró así: el $55 \%$ intermediación financiera; el $27 \%$, explotación de minas y canteras; el $15 \%$, actividades inmobiliarias, empresariales y de alquiler y el $1 \%$, comercio al por mayor y al por menor y reparación de vehículos.
Cuadro 2. Valor de los activos de las empresas de Santafé según sector económico y tamaño.

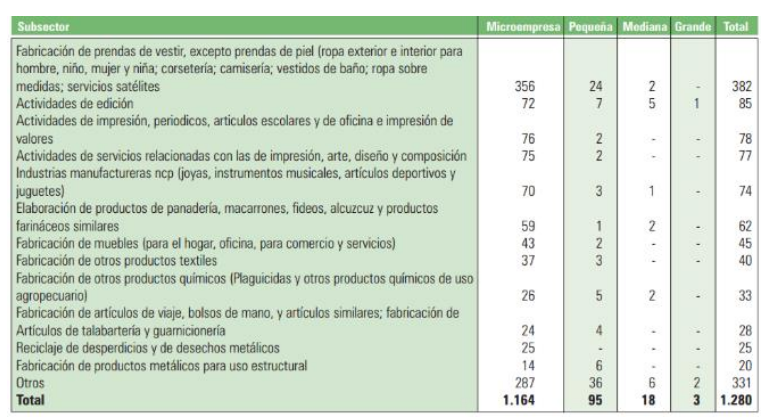

Fuentes: CCB (2006). Registro Mercantil, Cámara de Comercio de Bogotá. Proceso: Dirección de Estudios e Investigaciones de la CCB.

\section{Prácticas comerciales actuales de los microempresarios}

Las prácticas de mercadeo empleadas son pocas y cuando las desarrollan lo hacen sin un estudio previo lo cual, en términos generales, no genera buenos resultados:

- La relación con los proveedores es fijada por el precio y la calidad de los productos ofertados, sin embargo existe rotación de los mismos.

- El canal de distribución encontrado es el directo, no se manejan intermediarios en ninguno de los casos dado el tamaño de las empresas.

- En los diagnósticos existentes sobre las debilidades del empresario en la Pyme esta su escaso manejo del entorno, el desconocimiento de las posibilidades globales y de sus restricciones.

- A pesar de su trayectoria, la crisis económica las ha golpeado muy fuertemente, es así que cerca del 50 $\%$ de los empresarios manifiesta que sus ventas se han reducido y casi el $60 \%$ que sus utilidades han disminuido en los últimos dos años.

- En cuanto al perfil del empresario Pyme se encuentra que tiene amplia experiencia empresarial aunque no en el mismo negocio, casi las tres cuartas partes de los mismos tienen más de diez años gestionando empresa, lo que evidencia su espiritu emprendedor.

- Valdría la pena adentrarse en el conocimiento de este tipo de empresarios para identificar lo que lo promueve y formular con base en ello los programas que incentiven la creación de empresas. Más del 70 $\%$ de los dueños de las Pymes en Colombia han 
adelantado estudios superiores, aunque no se puede establecer si finalizados o no, y la mayoría son gerentes jóvenes, entre los 25 y 45 años, por lo que tienen todo un camino por delante que recorrer.

Las prácticas de distribución son mínimas y delegadas a terceros:

- $\quad$ En general mayoristas de la misma zona, la localidad es rica en sectores de mayoreo donde los pequeños comerciantes se abastecen. Puede ser una buena alternativa ya que se convierten en apoyo para la cadena de distribución general pero aumentando sustancialmente el valor final del producto.

- La localidad se abastece de los San Andresito y san Victorino, para algunos productos, no existe una logística integrada, los empresarios compran en la medida de sus necesidades y obtienen descuentos por cantidad y por fidelidad.

- Las empresas de restauración se abastecen semanalmente en plazas de mercado de la zona, tipo Paloquemao. Para productos perecederos tiene un mayorista el cual distribuye a diario según solicitud.

- Carecen de herramientas tecnológicas, no existen dispositivos electrónicos que permitan mantener pedidos máximos y mínimos.

- Solo algunos sectores tipo metalurgia y metal mecánica gozan de flota de transporte propia, los demás el abastecimiento es empírico y en general se usan métodos tales como transporte urbano 0 vehículos particulares.

- En abarrotes el abastecimiento es diario y depende de grandes compañías de alimentos que hacen rondas una a dos veces por semana para realizar las solicitudes de pedido.

- Los niveles de inventarios son mínimos, ya que se carece de capital de stock, existe un amplio desconocimiento de políticas serias de almacenamiento e inventarios.

- Existen procesos mínimos de transformación, por lo general se trata de producto terminado importado y en muchas ocasiones ingresado al país de manera ilegal, el mercado mayorista se caracteriza por la alta concentración de carteles y el contrabando que abastece los micro comercios de la zona.

- $\quad$ Si bien existen planes de ordenamiento comercial, el proceso es lento y desordenado, aún se mantienen muchos brotes de comercio informal que se abastecen día a día en estos mismos puntos. El modelo de suministro obliga al manejo de dinero en efectivo y el poco uso de apoyos comerciales por parte del distrito.

- $\quad$ Existe carencia y desconocimiento total del concepto promesa de servicio, las ventas están más atadas a la fidelidad y al precio para algunos sectores, si bien el concepto promesa de servicio es ambiguo si existen paramentos que permitan medirlo.

- Existe una pequeña frase que dice: "lo que no se mide, no se controla y no se puede mejorar". Las encuestas arrojaron total desconocimiento de indicadores de gestión, organización y distribución.

En cuanto al diagnóstico de las prácticas contables actuales que llevan a cabo los microempresarios evaluados se puede afirmar de manera agregada lo siguiente:

- Todos los microempresarios entrevistados tienen manejo de libro fiscal, sin conocimiento de su nombre real, ni de su significado e implicaciones.

- Solo dos de los encuestados reciben servicios de contador para el manejo de información con fines tributarios (anualmente para la declaración y pago del impuesto de renta, principalmente).

- Desconocimiento generalizado de las normas contables que les son aplicables.

- Confusión entre los requerimientos para su negocio en materia contable y en materia tributaria.

- Desconocimiento generalizado del proceso de convergencia hacia estándares internacionales de información financiera y lo correspondiente que se ha sido legislado en el país aplicable a las microempresas.

En general los microempresarios ven de manera muy positiva los lazos que se puedan crear para fortalecer su actividad empresarial con apoyo de la academia, de hecho coinciden en afirmar que su cercanía con los estudiantes de diversas áreas les permite recibir constantemente retroalimentación sobre algunas prácticas y por lo tanto aprecian mucho los esfuerzos formales a los que se le pueda dar curso con el propósito de mejorar.

A continuación describimos una serie de estrategias que se consideran útiles en cada una de las áreas evaluadas y que apuntan al fortalecimiento social y empresarial que motivó el desarrollo de este trabajo, todas ellas están enmarcadas en la capacitación y en el acompañamiento que se les pueda ofrecer: 


\section{En materia de mercadeo:}

La comprensión de las dinámicas productivas y comerciales de las Pymes necesita un análisis un poco más específico en cuanto a la identificación de los tipos de redes existentes (asociatividad). Para ello es indispensable el apoyo del estado y agremiaciones que soporten dicho proceso.

El papel de la academia y las entidades estatales fortalecería la mejora de las prácticas comerciales a través de talleres y capacitaciones sobre elaboración e implementación de planes de mercadeo que les permita identificar las oportunidades de mercado, la mejora de productos o servicios, la fijación de precios en el mercado, las relaciones con los proveedores y clientes, la distribución física del producto y el servicio posventa.

Debe llevarse a cabo un proceso de actualización periódica de la información sobre la caracterización, creación, transformación y liquidación de las empresas, así como sus aportes a la economía nacional. Debe existir consenso en relación con el número de Pymes, pues ninguna entidad estatal dispone de información específica sobre el estrato Pyme.

\section{En materia de distribución:}

En la medida que exista la voluntad de crecer y mejorar, los empresarios de la localidad deberán enfocar parte de su capital humano y financiero en una buena cadena de distribución. En primera medida se deberá realizar una evaluación, esta evaluación obliga al análisis de todos los niveles que componen la cadena de distribución desde el proveedor hasta el cliente final, identificando debilidades y fortalezas de la cadena dependiendo el sector.

Si el negocio se trata de producto terminado se deberán realizar un Benchmarking que permita identificar los competidores incluso de talla mundial, seguramente se están perdiendo oportunidades de negocio real. Si se realizan pequeñas transformaciones 0 trasformaciones sustanciales es importante educar el factor humano en cada departamento y empezar a manejar stocks en materias primas que así lo requieran o incursionar en tecnologías que permitan abasteciendo inmediato.

Es importante de la mano de buenas prácticas de marketing llegar al cliente a través de campañas y no solo quedarse en la prestación de un buen servicio, la mayoría de negocios llevan años manteniendo el mismo nivel de ventas si crecer.

El problema del transporte: las encuestas demostraron que los comerciantes de la localidad prestan mínima atención al problema de transporte, en la medida que el volumen de ventas aumente así mismo aumentará la rotación del producto lo que obliga a la necesidad de invertir en medios de transporte, rutas, seguimiento eliminando los tiempos de recarga, tiempo ociosos, es decir el tiempo en el que el camión está quieto. Si se mantiene la política de no adquirir este tipo de activos se debe trabajar en la necesidad contractual o jurídica con empresas de este sector. En logística se manifiesta que a medida que crece la flota de transporte crece la empresa.

Se debe priorizar en la adquisición de medios tecnológicos de control de pedidos y manejos de inventarios, hoy en día muchos proveedores reconocidos proveen a sus clientes de métodos que les permita mantener comunicación constante con ellos así como de material visual y de exhibición según volumen de ventas.

Es importante identificar asociaciones, los comerciantes de la zona ven a su par como su enemigo, si bien es su competencia, pequeñas asociaciones permitirían facilitar procesos de distribución y suministro obteniendo reducciones en los costes de la cadena por ende mayor margen de utilidad.

Finalmente, las propuestas de mejora deben ir encaminadas al liderazgo, en definitiva las actividades de comercio están manejadas por personas por lo tanto se debe tener claro el objetivo, para esto es importante identificar un líder, identificar las competencias de las personas idóneas en la parte de suministro, delimitar la empresa a través de áreas y siempre estar dispuesto a un mejoramiento continuo. Se puede trabajar en dos frentes empresas de tipo medio las cuales deben realizar un plan de negocio y una propuesta de internacionalización y las micro pymes que gozan de poco capital por lo tanto recurso humano poco capacitado, estas requieren mayor apoyo por parte de la alcaldía local que puede vincular a través de las universidades del sector programas piloto en determinas empresas seleccionadas donde se realice un plan de trabajo por periodos de tiempo y seguimiento periódico implementando indicadores de gestión.

\section{En materia contable:}

Emplear un plan de capacitación (inducción 0 alfabetización) en las diferencias que existen entre las obligaciones contables y las obligaciones tributarias a cargo de las microempresas, las cuales son autónomas e independientes entre sí conforme lo ratifica la Ley 1314 de 2009; así como también proponer un esquema para la planeación y desarrollo del proceso de convergencia en torno al manejo de la contabilidad simplificada para microempresas, definición de políticas contables y en el tema relacionado con los reportes financieros que anualmente debe preparar y presentar de conformidad con las NIF según Decreto 2706 de 2012. 


\section{CONCLUSIONES:}

- En Colombia existe consenso en cuanto a la importancia de la Pyme en el desarrollo económico y su contribución al equilibrio social. Existen alrededor de 47.750 Pymes registradas que representan al menos el 9 por ciento del parque empresarial nacional, generan más del 70 por ciento del empleo y más del 50 por ciento de la producción bruta de la industria, el comercio y los servicios. A pesar de lo anterior, es crítica la deficiencia de información sobre este estrato empresarial. Rodríguez (2003).

- No existe información actualizada, validada y periódica sobre su caracterización, creación, transformación y liquidación de empresas, ni sus aportes a la economía nacional. Ni siquiera hay consenso en relación con el número de Pymes, pues ninguna entidad estatal dispone de información específica sobre el estrato Pyme.

- La consolidación de una empresa en Colombia es un proceso que dura no menos seis años, hecho que debe tenerse en cuenta en los programas de apoyo y fomento para este estrato empresarial si verdaderamente se espera consolidar este tipo de unidades empresariales; pues la mayoría de las pequeñas y medianas empresas en Colombia tienen seis o más años de haber sido creadas.

- La comprensión de las dinámicas productivas y comerciales de las Pymes necesita un análisis un poco más específico en cuanto a la identificación de los tipos de redes existentes.

- En el proceso de convergencia contable el papel de la academia debe ser protagónico para que, en desarrollo de su función de proyección e impacto social, pueda ofrecer acompañamiento y soporte para el fortalecimiento social y empresarial como uno de los objetivos principales que se traza con la formulación de los nuevos lineamientos para el manejo de la contabilidad en el país.

- No existen políticas claras en cuanto a las micro pymes, solamente aquellas que tienen características exportadoras gozas de un plan de trabajo, sin embargo hay una gran cantidad de empresas de orden local que puede tener opciones de crecimiento.

- Los programas de desarrollo empresarial son dependientes de la administración de turno, lo cual obliga cada 4 años a poner en marche nuevos proyectos de trabajo.
- La mayoría de micro empresarios incursionan en el negocio como medio de subsistencia es decir bajo capital de trabajo, o flujos de caja fluctuantes. Se podría casi que llamar comercio informal.

- Existe desconocimiento total por parte de los empresarios de incentivos y mecanismos que permitan regular la parte impositiva, los comerciantes actúan casi que clandestinos para evitar impuestos de orden distrital.

- No existe un censo real de comerciantes de la zona, muchos no tiene cámara de comercio, y muchos desaparecen en muy poco tiempo por falta de planificación e investigación, las cámaras de comercio de la cuidad no tiene vocación de apoyo al empresario y están totalmente des entendidas de los pequeños empresarios.

- Algunos sectores tales como el comercio al por mayor, la bisutería, la industria textilera, los abarrotes gozan de buena salud, sin embargo crecen de formas desordenada, es importante, agruparlos, organizarlos, estimularlos de forma tal que la cultura del todo se puede sea dejada de lado.

\section{REFERENCIAS:}

Cámara de Comercio de Bogotá (2007). Perfil económico y empresarial de Santa Fe. Recuperado de http://es.scribd.com/doc/57755969/PerfilEconomico-Santafe\#scribd

Consejo Técnico de la Contaduría Pública, CTCP (2012). Direccionamiento Estratégico del proceso de convergencia de las normas de contabilidad e información financiera y de aseguramiento de la información, con estándares internacionales.

Documento CONPES 3678 del 26 de julio de 2010.

Gómez, O., de la Hoz, A., \& de la Hoz, B. (2011).Armonización de las NIC/NIIF en las prácticas contables de entes emisores no financieros que cotizan en la Bolsa de Valores de Caracas, Venezuela. Contaduría Y Administración, 233, 149-175.

Gornik-Tomaszewski, S. K. (2010). Adopting IFRS.(cover story). CPA Journal, 80(3), 12 - 18.

Hoffman, D y otros. (2007). Principios de Marketing y sus mejores prácticas. 3ra ed. Thomson. Mexico 
Kotler, P y Armstrong, G (2008). Fundamentos de Marketing. 14ª ed. México: Mc Graw Hill.

Kotler, P y Lane, K (2003). Dirección de Marketing. $12^{\mathrm{a}}$ ed. México: Pearson Prentice Hall.

Lamb, C, Hair, J y Mc Daniel, C (2002). Marketing. 6a ed. México: Thomson.

Ley 1314 de 2009. Recuperado de http://www.confiam.com/Ley $\% 201314 \% 20 \mathrm{de} \% 20$ 2009.pdf

Lloch, J. (1993). Orientación al Mercado y Competitividad de la empresa. España: Gestión 2000.

Manzano, M. P. (2005). Accounting Harmonization in Latin America: Moving toward IFRS.Research in Accounting Regulation, 18, 281-282.

McCarthy, J. (1984). Basic Marketing: Un Enfoque Gerencial. 8a ed. Buenos Aires: El Ateneo.

Moreno, M (2005). Mercadeo Estratégico. $1^{a}$ ed. Bogotá: Universidad Nacional Abierta y a Distancia.

Malhotra N, (2004) Investigación de Mercados. México. Ed. Pearson, 4ta edición.

Ministerio de Industria, Comercio y Turismo.

Orozco, Arturo J. (1999): Investigación de Mercados. Concepto y Práctica: Colombia, Editorial Norma, 1999.

Peter, J y Olson, Paul (2005). Comportamiento del Consumidor y Estrategia de Marketing. $6^{\mathrm{a}}$ ed. México: Mc Graw Hill.

Rodríguez, Astrid Genoveva (2003). La Realidad de la Pyme Colombiana: Desafío para el Desarrollo. FUNDES INTERNACIONAL.

Stanton, William (2007). Fundamentos de Marketing. 14 ed. México: Mc Graw Hill.

Secretaría de Cultura, Recreación y Deportes. Observatorios de Culturas (2008). Localidad de Santa Fe. Ficha básica.

Secretaría Distrital de Planeación (2007). GUÍA DE BARRIOS Y UPZ POR LOCALIDAD. Bogotá.

Superintendencia de Sociedades. (14 de marzo de 2012). Circular Externa 115-000002. Recuperado el 17 de 4 de 2013, de http://www.supersociedades.gov.co/web/Ntrabajo /Convergencia_NIC-NIIF/Circular_Externa_115002_De_2012.pdf

Superintendencia de Sociedades. (9 de enero de 2013). Circular Externa 115-000001. Recuperado el 17 de abril de 2013, de http://www.kpmg.com/CO/es/lssuesAndlnsights/A rticlesPublications/Documents/Circular_Externa_ 115-001_De_2013\%20Supersociedades.pdf

Superintendencia de Sociedades. (14 de marzo de 2013). Circular Externa 115-000003. Recuperado el 17 de abril de 2014, de http://actualicese.com/normatividad/2013/03/14/ci rcular-externa-115-000003-de-14-03-2013/

Vásquez, A (2006). Marketing Social Corporativo: Una Nueva Estrategia de Desarrollo Comercial en España. Madrid: eumed.net.

Zikmund W (2003). Fundamentos de Investigación de Mercados. Ed. Thomson. 